ing is found $(2,12)$. This study shows that Hoogsteen base pairs can coexist with Watson-Crick base pairs and it reinforces the possibility that such DNA polymorphism may be involved in various biological phenomena.

REFERENCES AND NOTES

1. A. H.-J. Wang et al., Nature (London) 282, 680 (1979).

2. D. E. Pulleyblank, D. B. Haniford, A. R. Morgan, Cell 42, 271 (1985).
3. A. H.-J. Wang et al., Science 225, 1115 (1984).

4. G. Ughetto et al., Nucleic Acids Res. 13, 2305 (1985).

5. M. M. Van Dyke and P. B. Dervan, Science 225, 1122 (1984).

6. C. M. L. Low, H. R. Drew, M. J. Waring, Nucleic Acids Res. 12, 4865 (1984)

7. C. M. L. Low, R. K. Olsen, M. J. Waring, FEBS Lett. 176, 414 (1984).

8. W. A. Hendrickson and J. Konnert, in Biomolecular Structure: Conformation, Function and Evolution, R. Srinivasan, Ed. (Pergamon, Oxford, 1979), p. 43.

9. M. Windholz, Ed., The Merck Index (Merck and Co., Inc., Rahway, NJ, ed. 10, 1983).

10. K. A. Hartman, Jr., and A. Rich, J. Am. Chem. Soc. 87, 2033 (1965)
11. A. Rich, A. Nordheim, A. H.-J. Wang, Annu. Rev. Biochem. 53, 791 (1984)

12. H. R. Drew and R. E. Dickerson, EMBO J. 6, 663 (1982).

13. Supported by grants from the National Institutes of Health, National Science Foundation, American Cancer Society, Office of Naval Research, National Aeronautics and Space Administration, and the Netherlarids Organization for the Advancement of Pure Research. G.U. acknowledges support from NATO and Instituto Strutturistica Chimica, Consiglio Nazionale Delle Richerche (Italy). We thank T. Yoshida of Shionogi Co., Osaka, Japan, for the triostin A.

2 December 1985; accepted 18 March 1986

\section{Pertussis Toxin Gene: Nucleotide Sequence and Genetic Organization}

\section{CAMille LOCHT AND JeRRY M. KeITH}

The current pertussis vaccines, although efficacious, in some instances produce undesirable side effects. Molecular engineering of pertussis toxin, the major protective antigen, could provide a safer, new generation of vaccines against whooping cough. As a first critical step in the development of such a vaccine, the complete nucleotide sequence of the pertussis toxin gene was determined and the amino acid sequences of the individual subunits were deduced. All five subunits are coded by closely linked cistrons. A promoter-like structure was found in the $5^{\prime}$-flanking region, suggesting that the toxin is expressed through a polycistronic messenger RNA. The order of the cistrons is S1, S2, S4, S5, and S3. All subunits contain signal peptides of variable length. The calculated molecular weights of the mature subunits are 26,024 for $S 1$, 21,924 for $S 2,21,873$ for S3, 12,058 for S4, and 11,013 for S5. Subunits S2 and S3 share $70 \%$ amino acid homology and $75 \%$ nucleotide homology. Subunit S1 contains two regions of eight amino acids homologous to analogous regions in the A subunit of both cholera and Escherichia coli heat labile toxins.

$\mathrm{P}$ ERTUSSIS TOXIN ( 1 ) IS ONE OF THE various toxic components produced by virulent Bordetella pertussis, the microorganism that causes whooping cough. A wide variety of biological activities, such as histamine sensitization, insulin secretion, lymphocytosis promotion, and immunopotentiating effects can be attributed to this toxin (2). In addition, the toxin protects mice challenged intracerebrally $(3,4)$ or by aerosol (4) with virulent $B$. pertussis. Pertussis toxin is, therefore, an important constituent in the vaccine against whooping cough and is included in the acellular component vaccines being tested and used in several countries (5). However, the toxin may also be the cause of the harmful side effects associated with the current vaccines (6). It may be possible to develop a new vaccine with reduced side effects by genetic manipulation of the toxin gene.

The toxin is structured in a hexamer composed of five dissimilar subunits, designated $S 1$ through S5 relative to their electrophoretic migration in denaturing gels (7). Subunit $S 1$ contains an enzymatic adenosine diphosphate (ADP)-ribosylation activity
(8) and subunits S2 through S5 contain a target cell receptor binding activity (9). Thus, by analogy to other bacterial ADPribosylating toxins, pertussis toxin is structured in an A-B model $(7,10)$ in which the A moiety contains the enzymatic activity and the B moiety the binding activity (11).

The isolation of a 4.5-kb DNA fragment containing the pertussis toxin S4 subunit gene was described previously (12). Because of the polycistronic nature of prokaryotic genes, and by analogy to other A-B structured bacterial toxins that have similar enzymatic activities $(13,14)$, it seemed likely that the cistrons coding for all the pertussis toxin subunits would be linked. We therefore determined the nucleotide sequence of the 4.5-kb cloned fragment. Here, we present this sequence and demonstrate that it indeed codes for the entire pertussis toxin structural gene.

Digests with a variety of six base pair (bp)-specific restriction enzymes and DNA sequence analysis were used to establish the restriction map shown in Fig. la. DNA was sequenced by the base-specific chemical cleavage method (15) starting from the re- striction sites Eco RI, Sal I, Sma I, and Bgl II and by the dideoxy chain termination method (16) after subcloning overlapping regions into vectors $\mathrm{M} 13 \mathrm{mpl} 18$ and M13 mpl9 (17). Because of the high $\mathrm{C}+\mathrm{G}$ content of $B$. pertussis DNA, it was necessary to use both of these methods with a combination of $8 \%$ and $20 \%$ polyacrylamide- $8 M$ urea gels for sequence analysis. Each nucleotide has been sequenced in both directions an average of 4.13 times. The final consensus sequence of the sense strand is presented in Fig. 2. In agreement with the overall high $\mathrm{C}+\mathrm{G}$ content of $B$. pertussis DNA (18), the entire sequence contains $62.2 \% \mathrm{C}+\mathrm{G}$ with $19.6 \% \mathrm{~A}, 33.8 \% \mathrm{C}, 28.4 \% \mathrm{G}$, and $18.2 \% \mathrm{~T}$ in the sense strand.

The DNA sequence was translated in all six reading frames. On the basis of our previous data (12), the open reading frame (ORF) corresponding to the $\$ 4$ subunit was identified and is shown in Fig. Ib. The assignment of the other subunits to their respective ORF's, as shown in Fig. 1, b and c, is based on the following evidence: size of ORF's, high coding probability, deduced amino acid composition, predicted molecular weights, ratios of acidic to basic amino acids, amino acid homology to other bacterial toxins, mapping of Tn5-induced mutations, and partial amino acid sequence.

Those ORF's long enough to code for any of the five toxin subunits were analyzed by the statistical TESTCODE algorithm designed to differentiate between real protein coding sequences and fortuitous open reading frames (19). The amino acid composition of each ORF with a high protein coding probability was calculated, starting from either the predicted amino terminus of the mature proteins or from the first amino acid of the mature protein determined by amino acid sequencing of subunits purified by high-performance liquid chromatography. These data were then compared with

Department of Health and Human Services, Public Health Service, National Institutes of Health, National Institute of Allergy and Infectious Diseases, Laboratory of Pathobiology, Molecular Pathobiology Section, Rocky Mountain Laboratories, Hamilton, MT $\mathbf{5 9 8 4 0 .}$ 
the experimentally determined compositions of the individual subunits (7). On the basis of the similarity of the amino acid compositions shown in Table 1 , all five subunits were identified and assigned to the ORF regions shown in Fig. lb, and the gene order of S1, S2, S4, S5, and S3 from the 5'end to the $3^{\prime}$-end was established (Fig. 1d).

Table 1 shows that the deduced amino acid composition from all five assigned subunits are in good agreement with the experimentally determined compositions, with two significant exceptions. First, the S1 subunit contains no lysine residues in the deduced amino acid sequence, whereas a $2.2 \%$ lysine content was determined experimentally. Second, in subunits S2, S3, S4, and S5 the proportion of cysteines was substantially underestimated in the experimentally observed compositions. These discrepancies, as well as the remaining minor differences observed for all subunits, including the previously assigned S4 subunit, can only "be attributed to experimental error during amino acid analysis (20) or to the individual subunits, which are purified by liquid column chromatography, being slightly impure. The amino acid composition of significant ORF's (Fig. 1, b and c) not assigned to any subunit show no similarity to any of the experimentally determined amino acid compositions, although some of these ORF's are quite long and have a high coding potential. It is possible that these

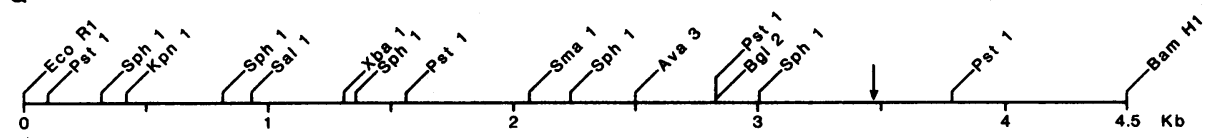

b

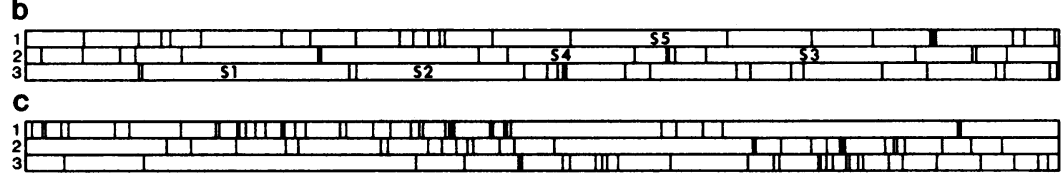

d

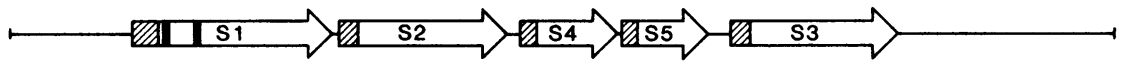

Fig. 1. Physical map and genetic organization of the pertussis toxin gene. (a) Restriction map of the 4.5$\mathrm{kb}$ Eco RI-Bam HI fragment from PPTX42 containing the pertussis toxin gene cloned from $B$. pertussis strain 3779 (12). The arrow indicates the position of the Tn5 DNA insertion in pertussis toxinnegative Tn5-induced mutant strains BP356 and BP357 $(12,23)$. (b) Open reading frames in the forward direction. (c) Open reading frames in the backward direction. The vertical lines indicate termination codons. (d) Organizational map of the pertussis toxin gene. The arrows show the translational direction and length of the protein coding regions for the individual subunits. The hatched boxes represent the signal peptides. The solid bars in SI represent the regions homologous to the A subunits in cholera and $E$. coli heat labile toxins (see Fig. 3).

regions code for other proteins that may be involved in the assembly or transport of pertussis toxin.

The experimentally estimated molecular weights and isoelectric points of the individual subunits were compared to the calculated molecular weights and ratios of acidic to basic amino acids of the putative proteins encoded by the ORF's. Table 1 shows that differences in these ratios reflect corresponding differences in the observed isoelectric points for each subunit, that is, the higher the acidic content, the lower the isoelectric point. The calculated molecular weights also correspond well to the experimentally determined values, with slight differences for the S1 (less than 10\%) and the S5 (about 15\%) subunits. These small differences are within acceptable limits for protein molecular weights determined by sodium dodecyl sulfate-polyacrylamide gel electrophoresis (SDS-PAGE).

Table 1. Comparison of the observed amino acid composition with the calculated composition from DNA sequences for mature pertussis toxin subunits. The data for the observed values are from Tamura et al. (7); see also (20). ND, not determined.

\begin{tabular}{|c|c|c|c|c|c|c|c|c|c|c|c|}
\hline \multirow[b]{2}{*}{ Item } & \multicolumn{2}{|c|}{$S 1$} & \multicolumn{2}{|c|}{ S2 } & \multicolumn{2}{|c|}{ S3 } & \multicolumn{3}{|c|}{ S4 } & \multicolumn{2}{|c|}{ S5 } \\
\hline & $\begin{array}{l}\text { Observed } \\
\text { values }\end{array}$ & $\begin{array}{l}\text { Calculated } \\
\text { values }\end{array}$ & $\begin{array}{c}\text { Observed } \\
\text { values }\end{array}$ & $\begin{array}{l}\text { Calculated } \\
\text { values }\end{array}$ & $\begin{array}{c}\text { Observed } \\
\text { values }\end{array}$ & $\begin{array}{l}\text { Calculated } \\
\text { values }\end{array}$ & $\begin{array}{l}\text { Exp. } 1 \\
\text { observed } \\
\text { values }\end{array}$ & $\begin{array}{c}\text { Exp. } 2 \\
\text { observed } \\
\text { values }\end{array}$ & $\begin{array}{l}\text { Calculated } \\
\text { values }\end{array}$ & $\begin{array}{c}\text { Observed } \\
\text { values }\end{array}$ & $\begin{array}{l}\text { Calculated } \\
\text { values }\end{array}$ \\
\hline $\begin{array}{l}\mathrm{M}_{\mathrm{r}} \\
\mathrm{A} / \mathrm{B}^{*}\end{array}$ & $28 \mathrm{kD}$ & $\begin{array}{c}26.0 \mathrm{kD} \\
1.3\end{array}$ & $23 \mathrm{kD}$ & $\begin{array}{l}21.9 \mathrm{kD} \\
0.89\end{array}$ & $22 \mathrm{kD}$ & $\begin{array}{l}21.9 \mathrm{kD} \\
0.83\end{array}$ & $11.7 \mathrm{kD}$ & & $\begin{array}{l}12.1 \mathrm{kD} \\
0.65\end{array}$ & $9.3 \mathrm{kD}$ & $\begin{array}{c}11.0 \mathrm{kD} \\
1.4\end{array}$ \\
\hline pI $\dagger$ & 5.8 & & 8.5 & & 8.8 & & 10.0 & 10.0 & & 5.0 & \\
\hline $\mathrm{Ala}$ & 10.6 & 11.5 & 6.5 & 6.0 & 11.7 & 11.1 & 9.4 & 9.8 & 8.2 & 9.8 & 9.0 \\
\hline $\mathrm{Arg}$ & 5.9 & 9.0 & 6.2 & 6.0 & 6.1 & 6.5 & 5.1 & 5.4 & 5.5 & 3.3 & 3.0 \\
\hline Asn $\neq$ & 9.3 & $\begin{array}{l}5.6 \\
43\end{array}$ & 6.3 & 2.5 & 6.3 & 2.0 & 5.3 & 5.0 & 0.9 & 8.2 & 3.0 \\
\hline $\begin{array}{l}\text { Asp } \\
\text { Cys }\end{array}$ & 1.0 & $\begin{array}{l}4.3 \\
0.9\end{array}$ & 1.3 & $\begin{array}{l}4.0 \\
3.0\end{array}$ & 1.1 & $\begin{array}{l}4.0 \\
3.0\end{array}$ & 0.9 & 0.7 & $\begin{array}{l}3.6 \\
3.6\end{array}$ & 1.6 & $\begin{array}{l}5.0 \\
4.0\end{array}$ \\
\hline Gln\$ & 10.6 & 3.0 & 8.7 & 3.5 & 9.0 & 4.5 & 9.5 & 9.1 & $\begin{array}{l}5.0 \\
3.6\end{array}$ & 9.3 & 3.0 \\
\hline Glu & & 7.3 & & 4.0 & & 3.5 & & & 4.5 & & 6.0 \\
\hline Gly & 11.2 & 7.7 & 13.0 & 10.6 & 11.9 & 10.1 & 9.6 & 8.9 & 6.4 & 8.7 & 8.0 \\
\hline His & 1.7 & 2.6 & 2.4 & 2.0 & 1.0 & 1.0 & 0.5 & 0.5 & 0.9 & 3.0 & 3.0 \\
\hline Ile & 3.2 & 3.4 & 4.2 & 5.5 & 5.0 & 6.5 & 2.0 & 1.8 & 1.8 & 3.4 & 3.0 \\
\hline Leu & 5.5 & 3.4 & 7.3 & 7.5 & 8.1 & 8.0 & 8.4 & 8.7 & 9.1 & 13.8 & 15.0 \\
\hline Lys & 2.2 & 0 & 3.4 & 3.0 & 2.7 & 2.5 & 6.9 & 7.6 & 7.3 & 4.7 & 5.0 \\
\hline Met & 1.6 & 1.7 & 1.4 & 1.5 & 1.1 & 1.5 & 5.1 & 4.3 & 7.3 & 1.6 & 2.0 \\
\hline Phe & 3.5 & 3.0 & 3.2 & 2.5 & 3.2 & 2.5 & 3.6 & 4.5 & 4.5 & 4.9 & 5.0 \\
\hline Pro & 4.4 & 3.4 & 4.6 & 4.5 & 5.7 & 5.0 & 9.1 & 9.9 & 10.0 & 5.6 & 5.0 \\
\hline Ser & 10.6 & 9.8 & 8.5 & 8.5 & 6.3 & 5.0 & 8.0 & 7.3 & 5.5 & 6.9 & 6.0 \\
\hline Thr & 7.4 & 7.3 & 10.4 & 10.1 & 8.2 & 8.0 & 5.0 & 5.1 & 4.5 & 6.9 & 7.0 \\
\hline $\operatorname{Trp}$ & ND & 0.9 & ND & 1.0 & ND & 0.5 & ND & ND & 0 & ND & 1.0 \\
\hline Tyr & 4.6 & 8.1 & 7.6 & 8.0 & 7.9 & 9.5 & 2.2 & 2.0 & 1.8 & 4.3 & 4.0 \\
\hline Val & 6.7 & 7.3 & 4.9 & 6.0 & 4.7 & 5.0 & 9.4 & 9.4 & 10.9 & 4.0 & 3.0 \\
\hline
\end{tabular}

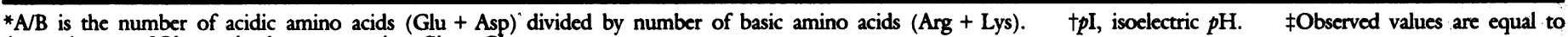
Asn + Asp. SObserved values are equal to Gin + Glu. 


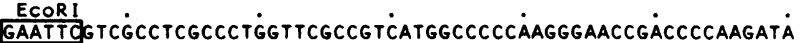
ATCGTCCTGCTCAACCGCCACATCAACGAGGCGCTGCAGTCCAAGGCGGTCGTCGAGGCC TTTGCCGCCCAAGGCGCCACGCCGGTCATCGGCCACGCCGGATCAGACCCGCGGCTTCATC GCAGACGAGÄTCCAGCGCT T 200 GCCGGCGTCGTGCGCGAAACCGGCGCCAAंGCTGAAGTAG CAGCGCAGCCCTCCAACGCGCCATCCCCGTCCGGCCGGCACCATCCCGCATACGTGTTGG CAACCGCCAÁCGCGCATGCG TGCAGATTCG TCGTACAAAACCCTCGATTCंTTCCGTACATं CCCGCTACTGCAATCCAACACGGCATGAACGCTCCTTCGGCGCAAAGTCGCGCGATGGTA

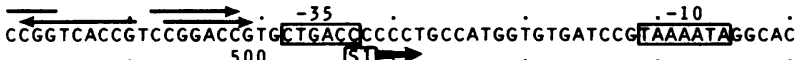
CATCAAAACGCAGAGGGGAAGACGGGATGCGTTGCACTCGGGCAATTCGCCAAACCGCAA $\begin{array}{llllllllllll}T & F M & R & C & T & R & A & I & R & Q & T & A \\ & & & & & & & & & \end{array}$ GAACAGGCTGGCTGACGTGGCTGGCGATTCTTGCCGTCACGGCGCCCGTGACTTCGCCGG CATGGGCCGACGATCCTCCCGCCACCGTATACCGCTATGACTCCCGCCCGCCGGAGGACG A W A $\because D$ D P P A T $V$ Y $R$ Y $D$ S R P P E A 700 D $V \quad F G Q N G G F$ T A W G N N D N V L D H H L T GACGTTCCTGCCAGGTCGGCAGCAGCAACAGCGCTTTCGICTCCACCAGCAGCAGCCGGC $G$ R S C Q V G S S N S A F V S T S S S R GCTATACCGAGGTCTATCTCGAACATCGCATGCAGGAAGCGGTCGAGGCCGAACGCGCCG

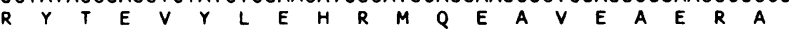
GCAGGGGCACCGGGCCACTTCATICGGCTACATCTACGAAGTCCGCGCCGACAACAATTTCT

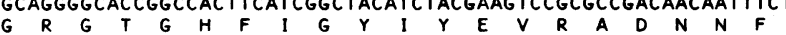
ACGGCGCCGCCAGCTCGTACTTCGAATACGTCGACACTTATGGCGACAATGCCGGCCGTA $\begin{array}{llllllllllllllllllll} & G & A & A & S & S & Y & F & E & Y & V & D & T & Y & G & D & N & A & G & R\end{array}$ TCCTCGCCGGCGCGCTGGCCACCTACCAGAGCGAATATCTGGCACACCGGCGCATTCCGC I L A G A L A T Y Q S E Y L A H R R I P CCGAAAACATICCGCAGGGTAACGCGGGTCTATCACAACGGCATCACCGGCGAGACCACGA $\begin{array}{llllllllllllllllllll}P & E & N & I & R & R & V & T & R & V & Y & H & N & G & I & T & G & E & T & T\end{array}$ CCACGGAGTÄTTCCAACGCTCGCTACGTCAGCCAGCAGACTCGCGCCAATCCCAACCCC

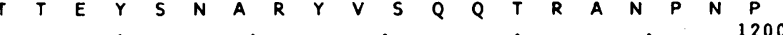
ACACATCGCGAAGGTCCGTAGCGTCGATCGICGGCACATTGGTGCATGGCGGCGGTGATA

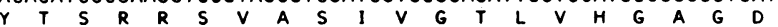

GCGCTTGCATGGCGCGGCAGGCCGAAAGCTCCGAGGCCATGGCAGCCTGGंTCCGAACGCG $\begin{array}{llllllllllllllllllll}S & A & C & M & A & R & Q & A & E & S & S & E & A & M & A & A & W & S & E & R\end{array}$ 1300

CCGGCGAGGCGATGGTTCTCGTGTACTACGAAAGCATCGCGTATTCGTTCTAGACCTGGC

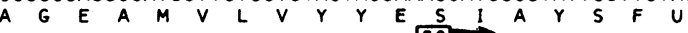

CCAGCCCCGCCCAACTCCGG TAATTGAACAGCATGCCGATCGACCGCAAGACGCTCTGCC ATCTCCTGTCCGTTCTGCCGTTGGCCCTCCंTGGGATCTCACGTGGCGCGGGCCTCCACGC $f M P$ I $D$ R K T L C H L L S V L P L A L L G S H V A.R A :S T CAGGCATCG $\dot{T}$ CATTCCGCCGCAGGAACAGÁTTACCCAGCATGGCAGCCCCंTATGGACGCT

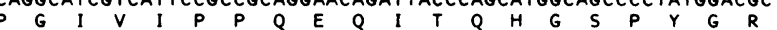
GCGCGAACAÄGACCCGTGCCंCTGACCGTGGCGGAATTGCGCGGCAGCGGCGATCTGCAGG $\begin{array}{llllllllllllllllllll}C & A & N & K & T & R & A & L & T & V & A & E & L & R & G & S & G & D & L & Q\end{array}$ 1600 AGTACCTGCG $\dot{T C A T G T G A C G C G C G G C T G G T C A A T A T T T G C G C T C T A C G A T G G C A C C T A T \dot{C}}$

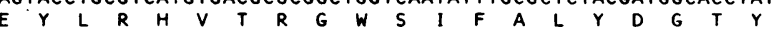
TCGGCGGCGAATATGGCGGं̈GTGATCAAGGACGGAACACCंCGGCGGCGCÄTTCGACCTGA

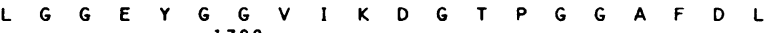
AAACGACGTTCTGCATCATGACCACGCGCAATACGGGTCAACCCGCAACGGATCACTACT K T T F $T$ C I M T T R N T G ACAGCAACG TCACCGCCACTCGCCTGCTCTCCAGCACCAACAGCAGGCTATGCGCGGTCT Y S N V T A T R L L S S T N S R L C A V

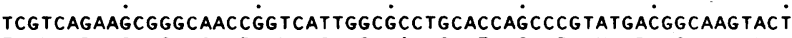

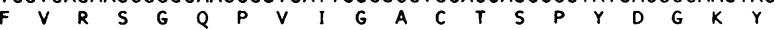
GAGCATGTACAGCCGGCTGCGGAAATGCTTACCTGOO 1900

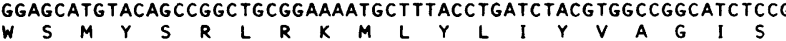
TACGCGTCCATGTCAGCAAGGAAGAACAG TATTACGACTATGAGGACGCAACGTTCGAGA $V R V H V$ S K E E Q Y Y D Y E D A T F E 2000 . 200 . CTTACGCCCTTACCGGCATCTCCATCTGCAATCCTGGATCATCCTTATGCTGAGACGCT

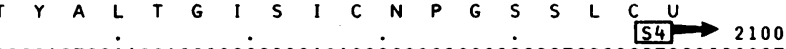
CCCCACTCGÁACCACCGCCंCCGGGACAGGGCGGCGCCCGGCGGTCGCGCGTGCGCGCCCT

Fig. 2. Complete nucleotide sequence of the pertussis toxin gene. The insert DNA from pPTX42, that is, the 4.5-kb Eco RI-Bam HI fragment shown in Fig. la, was digested with restriction enzymes and subcloned by standard procedures (43) with the cloning vectors M13 mpl8 and M13 mpl9 and $E$. coli strain JM10l (17). Both strands of the DNA were sequenced by either the Maxam and Gilbert base-specific chemical cleavage method (15) or the dideoxy chain termination method (16) with the universal 17-base primer, or both. The DNA sequence and the derived amino acid sequence were analyzed with the use of MicroGenie computer software. The deduced
GGCGTGGTTGCTGGCATCCGGCGCGATGACGCATCTTTCCCCCGCCCTGGCCGACGTTC.

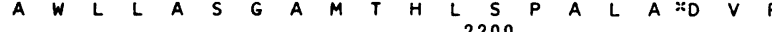
TTATGTGCTGGTGAAGACCAATATGGTGG TCACCAGCGTAGCCATGAAGCंCGTATGAAG $\begin{array}{llllllllllllllllllll}Y & V & L & V & K & T & N & M & V & V & T & S & V & A & M & K & P & Y & E & V\end{array}$ CACCCCGACGCGCATGCTGGTCTGCGGCATCGCCGCCAAACTGGGCGCCGCGGCCAGCAG $T$ P $T$ R $M$ M L V C C G I A A $K$ K L G A A A $S$ S CCCGGACGCGCACGTGCCGTTCTGCTTCGGCAAGGATCTCAAGCGTCCCGGCAGCAGTCC $\begin{array}{llllllllllllllllllllllllllll}P & D & A & H & V & P & F & C & F & G & K & D & L & K & R & P & G & S & S & P\end{array}$ CATGGAAGTCATGTTGCGCGCCGTCTTCATGCAACAACG $\dot{C} C$ CGCTGCGCAंGTTTCTGGG $M E V M L R A V F M Q Q R P R$ TCCCAAGCAÄCTCACTTTC $\dot{G A A G G C A A G C C G G C G C T C G A A C T G A T C C G G A}$ TIGGTCGAATG

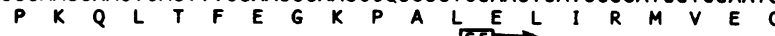
CAGCGGCAAGCAGGATTGCCCCTGAAGGCGAACCCCATGCATACCATCGCATCCATCCTG

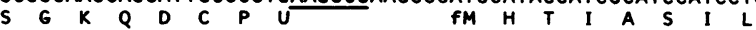
TTGTCCGTGCंTCGGCATATACAGCCCGGCTGACGTCGCCGGCTTGCCGACCCATCTGTAC

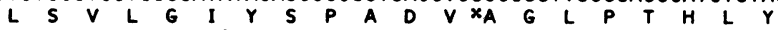
AAGAACTTCACTGTCCAGGAGCTGGCCTTGAAACTGAAGGGCAAGAATCAGGAGTTCTGC $K$ N F T V Q E L A L K L K G K N Q E F C CTGACCGCCTITCATGTCGGG CAGAAGCCTGGTCCGGGCGTGCCTGTCCGACGCGGGACAC L T A F M S G R S L V R A C L S D A G H GAGCACGACACGTGGTTCGACACCATGCTTGGCTTTGCCATATCCGCGTATGCGCTCAAG E H D T W F D T M L G F A I S A Y A L K AGCCGGATCGंCGTTGACGGTGGĀAGACTCGCCGTATCCGGGCACTCCCGGCGATCTGCTC S R I A L T V E D S P Y P G T P G D T L L GAACTGCAGATCTGCCCGCTICAACGGATATTTGCGAATGAACCCTTCCGGÁGGTTTCGACG E L Q I C P L N G Y C E U

TTTCCGCGCAATCCGCTTGAGACGATCTTCCGCCCTGGTTCCATTCCGGGAACACCGCA. $\underset{\text { CATGCTGATCAACAACAAGAAGCTGCTTCATCACATTCTGCCCATCCTGGTGCTCGCCCT }}{3000}$ CATGCTGATCAACAACAAGAAGCTGCTTCATCACATTCTGCCCATCCTGGTGCTCGCCCT GCTGGGCATGCGCACGGCC CAGGCCGTTGC̈GCCAGGCATCGTCATCCCGCCGAAGGCACT $L$ G M R T A Q A XV A P G I V I P P K A L GTTCACCCAACAGGGCGGCGCCTATGGACGCTGCCCGAACGGAACCCGCGCCTTGACCG

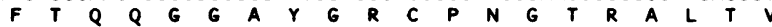
GGCCGAACTGCGCGGCAACGCCGAATTGCAGACGTATTTGCGCCAGATAACGCCCGGCTE

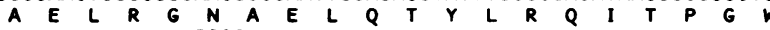
GTCCATATACGGTCTCTATGACGGTACGTACCTGGGCCAGGCGTACGGCGGCATCATCAA

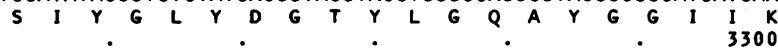
GGACGCGCCG CCAGGCGCGGGGTTCATTTATCGCGAAACTITTCTGCATCACGACCATATA

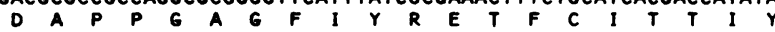

CAAGACCGGGCAACCGGCTGCGGATCACTACTACAGCAAGGTCACGGCCACGCGCCTGC

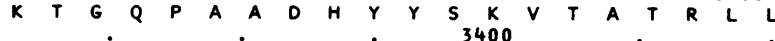
CGCCAGCACCAACAGCAGG $\dot{C}$ TGTGCGCG TATTCGTCAGGGACGGGCAATCGGTCATCG $\begin{array}{llllllllllllllllllllll}A & S & T & N & S & R & L & C & A & V & F & V & R & D & G & Q & S & V & I & G\end{array}$ AGCCTGCGCCAGCCCGTATGAAGGCAGGTACAGAGACATG'TACGACGCGCंTGCGGCGCCi $\begin{array}{llllllllllllllllllll}A & C & A & S & P & Y & E & G & R & Y & R & D & M & Y & D & A & L & R & R & L\end{array}$ GCTGTACATGATCTATATGTCCGGCCTTGCंCGTACGCGTCICACGTCAGCAAGGAAGAGCA

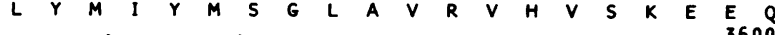
GTATTACGACTACGAGGACGCCACATTCCAGACCTATGCCCCTCACCGGCATTTCCCTCTG $\begin{array}{llllllllllllllllllll} & Y & D & Y & E & D & A & T & F & Q & T & Y & A & L & T & G & I & L & C\end{array}$ CAACCCGGCAGCGTCGATATGCTGAGCCGCCGGCTCGGATCTGTTCGCCTGTCCATGTTT N P A A S I C U

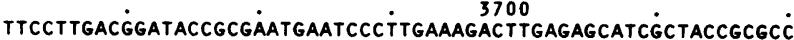

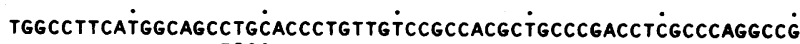
GCGGCGGGCTGCAGCGCTGTCAACCACTTCATGGCGAGCATCGTGGTCGTACTGCCGCGE CGGTCAGTGGCCACGGTGACCATCGCCATAATCTGGGCGGGCTACAAGCTGCTGTTCCGG CACGCCGATGTTGCTGGACGTGGTGCGTGTGGTGCTGGCGGGAGCTGCTGÄTCGGCGCATC GGCCGAAATCGCTCGTTATCTGCTGACCTGAATCCTGGACGTATCGAACATGCGTGATCC

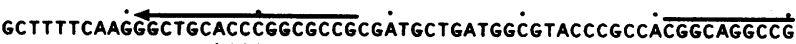
TGTGCAGCCGGCACCATTCCCTGCTGGGCCATCTCGGTTCAGCATCCGCTTTCTGGCCT' GTTTCCCGTGGCATTGCTGGCGATGCGGATCATGATCCGGGGCGATGACCAGCAGTTCCG CCISATA

amino acid sequence of the individual subunits are shown in the single letter code below the nucleotide sequence. The proposed signal peptide cleavage sites are indicated by asterisks. The start of the protein coding region for each subunit is indicated by the box and arrow over the initiation codon. Putative ribosomal binding sites are underlined. The promoter-like sequence is shown in the -35 and -10 boxes. Proposed transcriptional start site is indicated by the arrow in the CAT box. Inverted repeats are indicated by the arrows in the flanking regions. 
The assignment of $S 1$ is further supported by a significant homology of two regions in the amino acid sequence with two analogous regions in the A subunits of both cholera and Escherichia coli heat labile toxins (Fig. 3). These regions may be part of functional domains responsible for the similar catalytic activities of all three toxins (21). Furthermore, the assignment of $S 1$, as well as the correct prediction of the signal peptide cleavage site, is supported by preliminary amino acid sequence data for the mature protein (22). The absence of lysine residues in S1 may explain why lysine-specific chemical modifications do not affect the biological and enzymatic activities of $\mathrm{Sl}$ (10).

Subunits S2 and S3 share 70\% amino acid homology, which makes the correct assignment of these subunits to their ORF's diffcult if it is based only on the amino acid composition and the molecular weight. Nevertheless, we could determine the gene order as shown in Fig. Id with the help of a Tn5-induced $B$. pertussis mutant. Although unable to produce active pertussis toxin in the culture supernatant (23), this mutant still produces the $S 2$ subunit (24), thus the mutation is not located in the cistron for S2. We previously mapped this Tn5-insertion $1.3 \mathrm{~kb}$ downstream from the start site for the $S 4$ subunit cistron (12), which positions it in the ORF for the $S 3$ subunit (Fig. 1).

The amino acid sequence for each subunit was deduced from the nucleotide sequence and is presented in Fig 2. The mature proteins contain 234 amino acids for $S 1$, 199 amino acids for $S 2$ and S3, 110 amino acids for S4, and 100 amino acids for S5. The calculated molecular weights from the deduced sequence of the mature peptides are 26,024 for $S 1,21,924$ for $S 2,21,873$ for S3, 12,058 for $\$ 4$, and 11,013 for S5. Since S4 is present in two copies per toxin molecule $(7,10)$, the total molecular weight for the holotoxin should be 104,950. This is in agreement with the apparent molecular weight estimated by nondenaturing PAGE.

It is likely that all subunits contain signal peptides, as expected for secretory proteins. The length of the putative signal peptides was estimated after analysis of the hydrophobicity plot (25), the predicted secondary structure (26), and application of von Heijne's rule for the prediction of the most probable signal peptide cleavage site (27). The predicted cleavage site for each subunit is indicated in Fig. 2 by the asterisks and has been confirmed. for S1, S3, and S4 by sequencing the amino termini of the purified mature subunits $(12,22)$. The length of the signal peptides varies from 34 residues for $S 1,28$ residues for $S 3$, and 27 residues for $S 2$, to 21 residues for $S 4$, and 20 resi-

\section{Region 1}

Pertussis S1 subunit

Cholera A subunit

E. coli HLT A subunit

(8) Tyr Arg Tyr Asp Ser Arg Pro Pro (15)

(6) Tyr Arg Ala Asp Ser Arg Pro Pro (13)

(6) Tyr Arg Ala Asp Ser Arg Pro Pro (13)

\section{Region 2}

Pertussis S1 subunit

Cholera A subunit

E. coli HLT A subunit

Fig. 3. Comparison of two homologous regions in ADP-ribosylating subunits of pertussis, cholera, and $E$. coli heat labile toxins. The numbers in parentheses refer to the amino acid position in the mature proteins. Escherichia coli heat labile and cholera toxin sequences are from Yamamoto et al. (21). Common residues in all three toxins are boxed. HLT, heat labile toxin.

dues for S5. All of the signal peptides contain a positively charged amino terminal region of variable length, followed by a sequence of hydrophobic amino acids, usually in $\alpha$-helical or partially $\alpha$-helical, partially $\beta$-pleated conformation. A less hydrophobic carboxyl terminal region follows, usually ending in a $\beta$-turn conformation at the signal peptide cleavage site. All subunits except $S 5$ follow the $-1,-3$ rule (28), which positions the cleavage site after Ala-XAla. The amino terminal charge for the subunit signal peptides varies between +4 for $\mathrm{Sl}$ and +1 for S4 and S5. All described properties correspond very well to the general characteristics for bacterial signal peptides (29).

An interesting feature of the sequence is the overall, relatively high amount of cysteines compared to $E$. coli proteins. Cysteines are not involved in inter-subunit links in the quaternary structure of the toxin, since all subunits can be separated by SDSPAGE in the absence of reducing agents. The cysteines are probably involved in intrachain bonds, since reducing agents signifcantly change the electrophoretic mobility of all subunits except $S 4$ (30). Serines, threonines, and tyrosines also are represented more frequently than in average $E$. coli proteins. The hydroxyl groups of these residues may be involved in the quaternary structure through hydrogen bonding.

The most striking feature of the predicted peptide sequences is the high degree of homology between S2 and S3. The two peptides are $70 \%$ homologous in their amino acid sequences and $75 \%$ homologous in their nucleotide sequences. This suggests that both cistrons were generated through a duplication of an ancestral cistron followed by mutations that result in functionally different peptides. Despite their high homology, also reflected in the predicted secondary structures and hydrophilicities, S2 and S3 subunits cannot substitute for each other in the functionally active pertussis toxin (7). The comparison between the two subunits may be useful in localizing their functional domains in relation to their primary, secondary, and tertiary structures. The differ- ences between S2 and S3 are scattered throughout the whole sequence and are slightly more frequent in the amino terminal half of the peptides. Each of the subunits binds to one $\$ 4$ subunit (7). This function could be located in the more conserved carboxyl terminal domains of S2 and S3. The two resulting dimers are thought to bind to the same S5 subunit. This function could be assigned to the more divergent amino terminal domains of S2 and S3. Alternatively, it is possible that the dimers bind to the S5 subunit through S4 and that the amino terminal domains of $S 2$ and $S 3$ are involved in some other function, possibly the interaction of the binding moiety (S2 through S5) with the enzymatically active moiety (S1).

The $S 1$ subunit was compared to the $A$ subunits of other bacterial toxins. Two regions with significant homology to cholera and $E$. coli heat labile toxins were found (Fig. 3). They are located in analogous regions of all three toxins. However, the three amino acid differences found in these regions cannot be explained by single base pair changes in the DNA. Furthermore, in most cases the homologous amino acids use quite different codons in pertussis toxin compared to cholera and $E$. coli heat labile toxins. This, together with the fact that no other significant homology in the primary structure could be found and that the amino acid sequences of the other subunits are completely different from the sequence of any other ADP-ribosylating toxin, strongly suggests that pertussis toxin is not evolutionarily related to any of the other known bacterial toxins. The nicotinamide adenine dinucleotide (NAD)-binding site for the two enterotoxins has been identified at the carboxyl terminal region of their $A_{1}$ subunit $(13,31)$. No significant homology could be found between the carboxyl terminus of the enterotoxins nor any other NAD-binding enzymes and the analogous region in the S1 subunit. This suggests that the NAD-binding function of the ADP-ribosylating enzymes is dependent more on the secondary or tertiary structures than on the primary structures. We propose that the two enzy- 
matically active domains lie in different regions of the protein, one at the amino terminal half of the subunit for the acceptor substrate $(\mathrm{Ni})$ binding and the other at the carboxyl terminal half of the subunit for the donor substrate $\left(\mathrm{NAD}^{+}\right)$binding.

Since all pertussis toxin subunits are closely linked and probably expressed in a very precise ratio, it is possible that they are arranged in a polycistronic operon. A polycistronic arrangement for the subunit cistrons also has been described for other bacterial toxins bearing similar enzymatic functions, such as diphtheria, cholera, and $E$. coli heat labile toxins $(14,21,32)$. We therefore analyzed the flanking regions for the presence of transcriptional signals. In the 5 '-flanking region, starting at position 469 , the sequence TAAAATA was found, which matches six of the seven nucleotides found in the ideal TATAATA Pribnow or -10 box. An identical sequence can be found in several other bacterial promoters, including the phage $\lambda$ L57 promoter (33). Given the fact that most transcripts start at a purine residue about five to seven nucleotides downstream from the Pribnow box, we tentatively located the transcriptional start site at the adenine residue at position $\mathbf{4 8 2}$. This residue is located in the sequence CAT, often found at transcriptional start sites (33). Upstream from the proposed -10 box, the sequence CTGACC starts at position 442 . This sequence matches four of the six nucleotides found in the ideal $E$. coli -35 box TTGACA. The mismatching nucleotides in the proposed pertussis toxin -35 box are the two end nucleotides, of which the $3^{\prime}$-residue is the less important nucleotide in the $E$. coli -35 consensus box. A replacement of the $T$ by a $C$ in the first position of the consensus sequence can also be found in several $E$. coli promoters (33). The distance between the two proposed promoter boxes is 21 nucleotides, a distance too long to be efficiently functional in $E$. coli, although a distance of the same length has been found in the galPl promoter (34) and in several plasmid promoters (33). The proposed -35 box is immediately preceded by two overlapping, short inverted repeats with calculated free energies (35) of $-15.6 \mathrm{kcal}$ and $-8.6 \mathrm{kcal}$, respectively. Inverted repeats can also be found at the $5^{\prime}$-end of the cholera toxin promoter. In both cases they may be involved in positive regulation of the toxin promoters. Evidence for a positive regulation of toxin biosynthesis in $B$. pertussis came through the discovery of the pir gene, the product of which is essential for the expression of many virulence factors, including pertussis toxin (36). None of the ORF's assigned to the other subunit is closely preceded by a similar promoter-like structure.

The 3 '-flanking region has been examined for the presence of possible transcriptional termination sites. Several inverted repeats could be found: the most significant is located in the region extending from position 4031 to 4089 and has a calculated free energy (35) of $-41.4 \mathrm{kcal}$. None of the inverted repeats are immediately followed by an oligo(dT) stretch, which may suggest that they function in a rho-dependent fash-

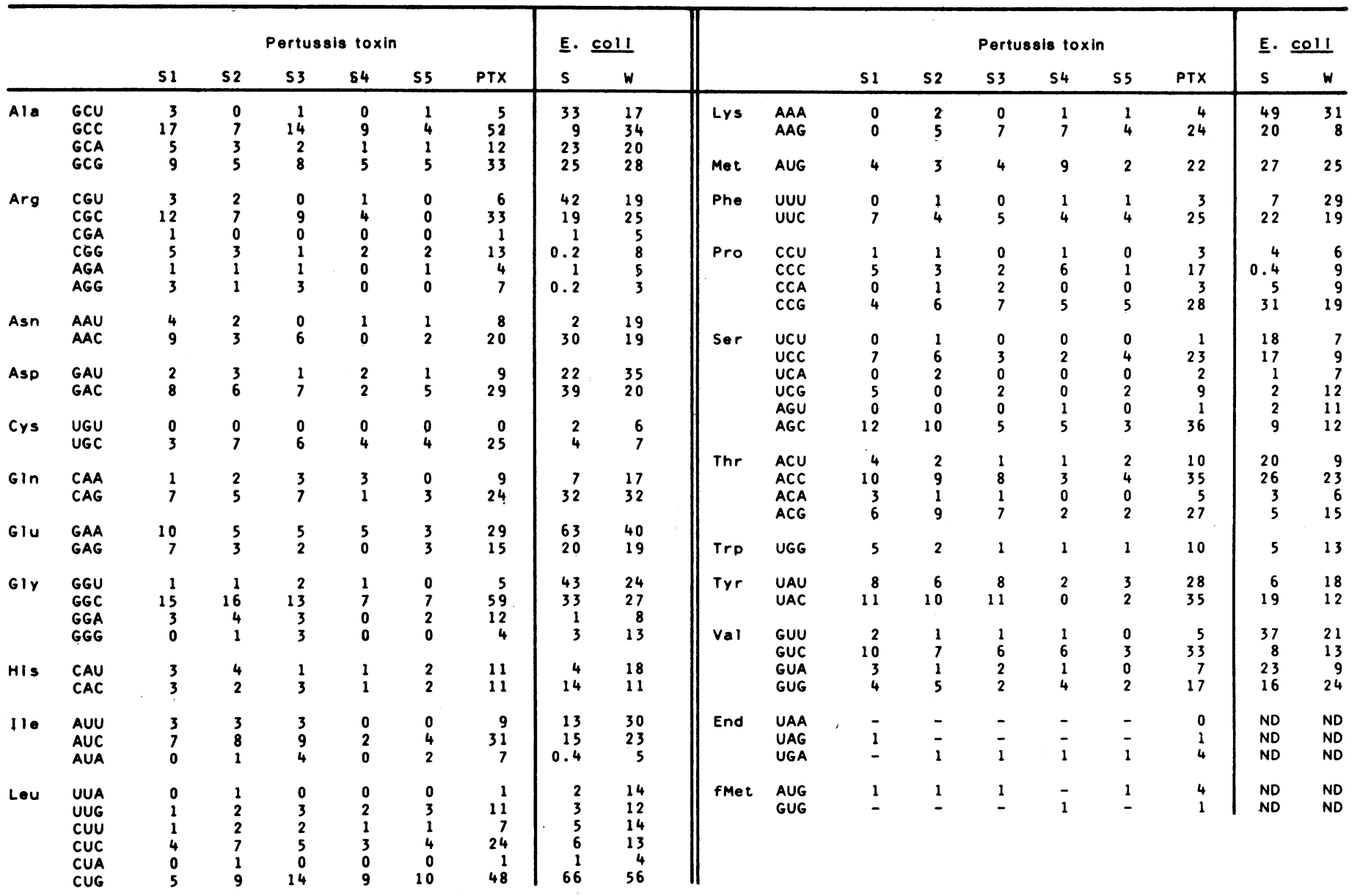

Fig. 4. Comparison of codon usage between pertussis toxin and strongly and weakly expressed $E$. coli genes. Absolute codon usage for the subunit cistrons of pertussis toxin include the signal peptides. The number of codons in the five individual subunits are $269(S 1), 227(S 2), 228(S 3), 132(S 4)$, and $121(S 5)$. The codon frequencies for $E$. coli are deduced from Grosjean and Fiers (42). S, strongly expressed genes; W, moderately to weakly expressed genes. All $E$. coli and the PTX data are expressed in relative codon usage per 1000 codons. The pertussis usage is based on 977 codons for the pertussis toxin gene (PTX). Escherichia coli usage is based on 5253 codons for highly expressed genes $(S)$ and 5231 codons for moderately to weakly expressed genes $(\mathrm{W})$. ND, not determined. 
ion (37). Whether any of these inverted repeats is functional in $B$. pertussis remains to be established and can be investigated by a small deletion or by site-directed mutagenesis experiments, which are feasible now that the DNA sequence is known. However, the gene may contain other, not yet identified, cistrons related to expression and possibly involved in regulation, assembly, or transport of functionally active pertussis toxin or other virulence factors. Nonstructural proteins involved in the posttranslational processing of $E$. coli heat labile toxin have been proposed (38). However, no significantly long ORF was found at the 3 '-end of the nucleotide sequence shown in Fig. Ib. If other proteins are encoded by the same polycistronic operon, their coding regions must be located further downstream.

Finally, we examined the $5^{\prime}$-flanking region of each cistron for the presence of ribosomal binding sites. Neither the ribosomal binding sequences for $B$. pertussis genes, nor the 3 '-end sequence of the $16 S$ ribosomal RNA (rRNA) are known. Therefore, we could only compare the flanking regions with the ribosomal binding sequences of heterologous prokaryotic organisms represented by the Shine-Dalgarno sequence (39). Preceding the $S I$ initiation codon we found the sequence GGGGAAG, starting at position 495 . This sequence shares four out of seven nucleotides with the ideal Shine-Dalgarno sequence AAGGAGG. The two first mismatching nucleotides in the pertussis toxin gene would not destabilize the hybridization to the 3 '-end of the $E$. coli $16 S \mathrm{rRNA}$. This putative ribosomal binding site is close enough to the initiation codon for $S 1$ to be functional in $E$. coli. Another possible Shine-Dalgarno sequence overlaps the first one and also matches four out of seven nucleotides to the consensus sequence. The mismatching nucleotides, however, have a more destabilizing effect than the ones found in the first sequence. The $S 2$ subunit ORF is not closely preceded by a ribosomal binding sequence, which may suggest that $S 2$ is translated through a mechanism not involving the detachment and reattachment of the ribosome between the coding regions for $\mathrm{Sl}$ and S2. The short distance between the S1 and $S 2$ cistrons and the absence of a ribosomal binding site are characteristic of this mechanism. A possible ribosomal binding site for S4 is the sequence CAGGGCGGC, starting at position 2066.The ORF for S5 is preceded by the sequence AAGGCG, starting at position 2485, which matches five out of six nucleotides in the consensus sequence AAGGAG. Finally, S3 is preceded by the sequence GGGAACAC, which is very similar to the proposed ribosomal binding site for S1, that is, GGGAAGAC.

Two different initiation codons (40) are used for the translation of all subunits in $B$. pertussis, that is, the most frequently used ATG for $S 1, S 2, S 3$, and S5, and the less frequently used GTG for S4. The initiation codon GTG is also found in the diphtheria toxin gene (14).

Attempts to efficiently express the pertussis toxin gene in $E$. coli have been unsuccessful thus far (12), although very sensitive monoclonal and polyclonal antibodies are available (24). This lack of expression in $E$. coli may be because $B$. pertussis promoters are not efficiently recognized by the $E$. coli RNA polymerase. Analysis of the promoter-like structure of the pertussis toxin gene and comparison of this structure to strong $E$. coli promoters reveal very significant differences indeed, of which the most striking one is the unusually long distance between the proposed -35 and -10 boxes in the pertussis toxin promoter. The distance between those two boxes in strong $E$. coli promoters is around 17 nucleotides (41), whereas the distance in the putative pertussis toxin promoter is 21 nucleotides. By using expression vectors designed to detect heterologous expression signals that are able to function in $E$. coli, we have found further evidence that $B$. pertussis promoters may not be recognized by the $E$. coli expression machinery.

Even in the presence of $E$. coli promoters, such as the inducible strong lac/trp fusion promoters, recombinant expression may be hampered by translational difficulties. Indeed, the codon usage (Fig. 4) is unsuitable for efficient translation of the pertussis toxin gene in $E$. coli (42). This is reflected by the codon choice for frequently used amino acids, such as alanine, arginine, glycine, histidine, lysine, proline, serine, and valine. Efficient expression in $E$. coli may require resynthesis of the pertussis toxin operon, respecting the optimal codon usage for $E$. coli. It is not known whether the codon usage for pertussis toxin reflects the optimal codon usage for expression in $B$. pertussis, since no other $B$. pertussis gene has been sequenced. Whether pertussis toxin is a strongly or weakly expressed protein in $\boldsymbol{B}$. pertussis and whether this expression is regulated by the presence of a precise relative amount of the different transfer RNA isoacceptors, possibly different from $E$. coli, can be evaluated by in vitro translation with the use of $E$. coli and $B$. pertussis cell-free extracts.

The cloned and sequenced pertussis toxin gene will facilitate the development of an efficient and safer vaccine against whooping cough. By comparison to other toxin genes with similar biochemical functions, and by physical identification of the active sites either for the ADP-ribosylation in the S1 subunit or the target cell binding in subunits S2 through S4, it is now possible to modify those sites by site-directed mutagenesis of the $B$. pertussis genome. These modifications could abolish the pathobiological activities of pertussis toxin without hampering its immunogenicity and protectivity. Alternatively, by knowing the DNA sequence it will be possible to map protective epitopes. Synthetic oligopeptides that include those epitopes also will be useful in the development of a new generation of vaccines.

\section{REFERENCES AND NOTES}

1. Pertussis toxin is also known as lymphocytosispromoting factor, leukocytosis-promoting factor, histamine-sensitizing factor, islets-activating protein, and pertussigen [for a review of pertussis vaccines, see C. R. Manclark and J. L. Cowell, in Bacterial Vaccines, R. Germanier, Ed. (Academic Press, New York, 1984), pp. 69-106].

2. J. J. Munoz, in Pertussis Taxin, R. D. Sekura, J. Moss, M. Vaughan, Eds. (Academic Press, New York, 1985), pp. 1-18.

3. J. J. Munoz, H. Arai, R. L. Cole, Infect. Immun. 32, 243 (1981).

4. H. Sato and Y. Sato, ibid. 46, 415 (1984).

5. Y. Sato, M. Kimura, H. Fukumi, Lancet 1984-I, 122 (1984).

6. L. Steinman et al., Proc. Natl. Acad. Sci. U.S.A. 82, 8733 (1985).

7. M. Tamura et al., Biochemistry 21, 5516 (1982).

8. T. Katada and M. Ui, J. Biol. Chem. 257, 7210 (1982); T. Katada, M. Tamura, M. Ui, Arch. Biochem. Bioplys. 224, 290 (1983).

9. M. Tamura, K. Nogimori, M. Yajima, K. Ase, M. Ui, J. Biol. Chem. 258, 6756 (1983).

10. M. Ui, K. Nogimori, M. Tamura, in Pertussis Taxin, R. D. Sekura, J. Moss, M. Vaughan, Eds. (Academic Press, New York, 1985), pp. 19-43.

11. D. M. Gill, in Bacterial Taxins and Cell Membranes, J. Jeljaszewics and T. Wadstrom, Eds. (Academic Press, New York, 1978), pp. 291-332.

12. C. Locht et al., Nucleic Acids Res. 14, 3251 (1986).

13. T. Yamamoto, A. Suyama, N. Mori, T. Yokota, A Wada, FEBS Lett. 181, 377 (1985); G. D. N. Pearson and J. J. Mekalanos, Proc. Natl. Acad. Sci. U.S.A. 79, 2976 (1982).

14. M. Kaczorek et al., Science 221, 855 (1983).

15. A. M. Maxam and W. Gilbert, Methods Enzymol. 65, 499 (1980).

16. F. Sanger, S. Nicklen, A. R. Coulson, Proc. Natl. Acad. Sci. U.S.A. 74, 5463 (1977).

17. J. Messing, Methods Enzymol. 101, 20 (1983).

18. K. Kersters et al., Int. J. Syst. Bacteriol. 34, 56 (1984).

19. J. W. Fickett, Nucleic Acids Res. 10, 5303 (1982).

20. In Tamura et al. (7), no error limits for the amino acid composition were given. However, since amino acids must be present in whole number molar ratios, it is easy to see that the percentage of His in S4, for example, is underestimated. In this mature subunit there is one His residue in 110 amino acids. Thus, the experimental value should be about $0.9 \%$, not $0.5 \%$. Operational factors contribute to errors in estimation of amino acid compositions. Such errors can be evaluated by including a known control protein in the analysis.

21. T. Yamamoto, T. Nakazawa, T. Miyata, A. Kaji, T. Yokota, FEBS Lett. 169, 241 (1984).

22. K. Marchitto, J. E. Coligan, J. M. Keith, unpublished results.

23. A. A. Weiss, E. L. Hewlett, G. A. Myers, S. Falkow, Infect. Immun. 42, 33 (1983).

24. K. Marchitto, J. J. Munoz, J. M. Keith, in preparation.

25. J. Kyte and R. F. Doolittle, J. Mol. Biol. 157, 105 (1982).

26. J. Garnier, D. J. Osguthorpe, B. Robson, ibid. 120, 97 (1978).

27. G. von Heijne, Eur. J. Biochem. 133, 17 (1983)

28. — J. Mol. Biol. 173, 243 (1984).

29. , ibid. 184, 99 (1985).

30. M. S. Peppler, R. C. Judd, J. J. Munoz, Dev. Biol. Stand., in press.

31. C. Y. Lai, Q. C. Xia, P. T. Salotra, Biochem. Bioploys. Res. Commun. 116, 341 (1983); L. K. Duffy, A. 
Kurosky, C. Y. Lai, Arch. Biochem. Bioplyys. 239, 549 (1985).

32. J. J. Mekalanos et al., Nature (London) 306, 551 (1983).

33. D. K. Hawley and W. R. McClure, Nucleic Acids Res. 11, 2237 (1983)

34. R. Musso, R. DiLauro, M. Rosenberg, B. deCrombrugghe, Proc. Natl. Acad. Sci. U.S.A. 74, 106 (1977).

35. I. Tinico et al., Nature (London) New Biol. 246, 40 (1973)
36. A. A. Weiss and S. Falkow, Infect. Immun. 43, 263 (1984)

37. S. Adhya and M. Gottesman, Annu. Rev. Biochem. 47, 967 (1978).

38. H. Hofstra and B. Witholt, J. Biol. Chem. 260 , 16037 (1985).

39. J. Shine and L. Dalgarno, Nature (London) 254, 34 (1975).

40. M. Kozak, Microbiol. Rev. 47, 1 (1983).

41. J. Brosius, M. Erfle, J. Storella, J. Biol. Chem. 260, 3539 (1985).
42. $H$. Grosjean and W. Fiers, Gene 18,199 (1982)

43. T. Maniatis, E. F. Fritsch, J. Sambrook, Molecular Cloning: A Laboratory Manual (Cold Spring Harbor Laboratory, Cold Spring Harbor, NY, 1982).

44. We thank Dr. K. Marchitto for reading the manuscript, S. Perryman for technical assistance, G. Hettrick for graphics, S. Smith for discussions, S. Smaus and $\mathrm{H}$. Blahnik for help in preparing the manuscript, and A. Maquet for her patience.

18 February 1986; accepted 8 April 1986

\section{Caffeine-Induced Uncoupling of Mitosis from the Completion of DNA Replication in Mammalian Cells}

\section{Robert Schlegel* and Arthur B. Pardee}

Caffeine was shown to induce mitotic events in mammalian cells before DNA replication (S phase) was completed. Synchronized BHK cells that were arrested in early $S$ phase underwent premature chromosome condensation, nuclear envelope breakdown, morphological "rounding up," and mitosis-specific phosphoprotein synthesis when they were exposed to caffeine. These mitotic responses occurred only after the cells had entered $S$ phase and only while DNA synthesis was inhibited by more than 70 percent. Inhibitors of protein synthesis blocked these caffeine-induced events, while inhibitors of RNA synthesis had little effect. These results suggest that caffeine induces the translation or stabilizes the protein product (or products) of mitosis-related RNA that accumulates in S-phase cells when DNA replication is suppressed. The ability to chemically manipulate the onset of mitosis should be useful for studying the regulation of this event in mammalian cells.

I N YEAST AND MAMMALIAN CELLS, MItosis does not occur when $S$ phase is interrupted by drug treatments or by restrictive conditions for mutants that are

Fig. 1. Cytoplasmic, chromosomal, and phosphoprotein synthesis changes in PCC-containing cells (indicated by arrows). Cells were plated at $1 \times 10^{5}$ per $60-\mathrm{mm}$ dish in Dulbecco's modified Eagle's medium (DME) +10 percent fetal calf serum (FCS) and incubated for 24 hours. Synchrony was achieved by incubation in isoleucinedeficient DME + 5 percent dialyzed FCS for 34 hours $(16,17)$. Cells were then treated with DME + 10 percent FCS $+2.5 \mathrm{mM}$ hydroxyurea for 14 hours, and finally were given the specified drugs $(T=0)$ along with Colcemid $(0.3 \mu \mathrm{g} / \mathrm{ml})$. (A) Phase contrast photograph $(\times 840)$ of "rounded up" PCC-containing cells 4 hours after the addition of $5 \mathrm{mM}$ caffeine. No "rounded up" cells were seen in untreated controls. (B) Condensed chromatin of cells $\mathbf{8}$ hours after the addition of $5 \mathrm{~m} M$ caffeine. Cells were fixed in absolute methanol and stained for 10 minutes with Hoechst $33242(1 \mu \mathrm{g} / \mathrm{ml})(\times 2100)$. (C) Chromosome preparation showing $S$-phase PCC stained with Hoechst $33242(\times 2100)$. Four hours after the addition of $5 \mathrm{mM}$ caffeine and Colcemid $(0.3 \mu \mathrm{g} / \mathrm{ml})$ the cells were trypsinized, treated for 20 minutes at $37^{\circ} \mathrm{C}$ in 0.55 percent $\mathbf{K C l}$, fixed for $\mathbf{3 0}$ minutes in methanol:acetic acid (3:1), dropped on wetted slides, and heated at $70^{\circ} \mathrm{C}$ overnight before staining. (D) Indirect immunofluorescence of mitosis-specific phosphoproteins present in both the cytoplasm and chromatin of the same PCC-containing cells shown in (B). Monoclonal antibody MPM-2 (9) and rhodamine-conjugated, goat-antimouse secondary. antibody were diluted $1: 500$ and $1: 1000$, respectively, in phosphate-buffered saline (PBS) con- temperature-sensitive for DNA synthesis (1). Although premature chromosome condensation (PCC) and breakdown of the nuclear envelope can be induced in S-phase
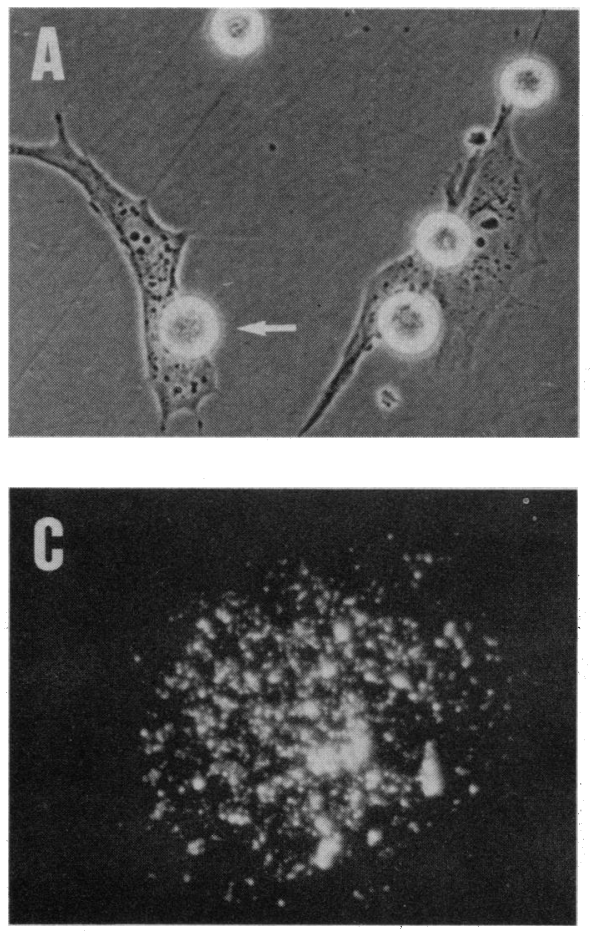

taining 1 percent bovine serum albumin. Methanol-fixed cells were preincubated at $37^{\circ} \mathrm{C}$ with $\mathrm{DME}+10$ percent FCS for 30 minutes; incubated with MPM-2 for 4 hours; rinsed successively cells by fusing them with mitotic cells $(2,3)$, these events result from the transfer of preformed mitotic factors, not the uncoupling of endogenously induced mitotic events from the completion of DNA replication. The BHK (Syrian hamster fibroblast) temperature-sensitive mutant tsBN2 $(4,5)$, which undergoes PCC and other early mitotic events at the restrictive temperature, provides the only example of a cell line in which mitotic events can occur before $S$ phase is completed. The biochemical processes that initiate mitosis are still unknown.

We have found that normal BHK cells that were arrested in $S$ phase (6) and treated with caffeine underwent, within a few hours, the same early mitotic events described by Nishimoto $(4,5)$ for the tsBN2 mutant. Caffeine could induce PCC at con-

Department of Pharmacology, Harvard Medical School and Division of Cell Growth and Regulation, DanaFarber Cancer Institute, Boston, MA 02115.

*To whom correspondence should be addressed at the Dana-Farber Cancer Institute.
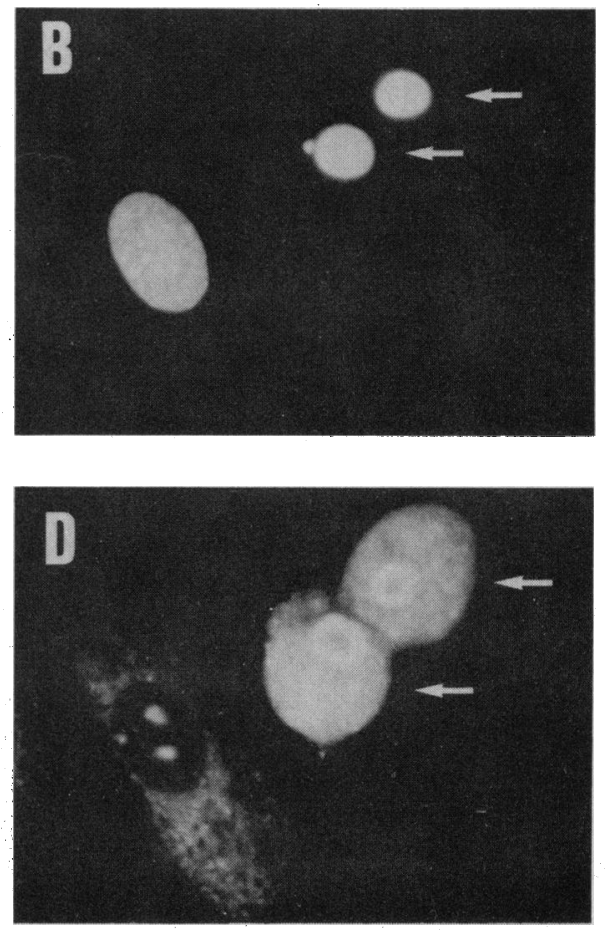

with DME + 10 percent FCS, PBS, and distilled water; air-dried; incubated with the fluorescent secondary antibody for 1 hour; and rinsed as before. 